\title{
QCD in terms of gauge-invariant dynamical variables
}

\author{
Hans-Peter Pavel* \\ Institut für Kernphysik, TU Darmstadt, D-64289 Darmstadt, Germany \\ Bogoliubov Laboratory of Theoretical Physics, JINR Dubna, Russia \\ E-mail: hans-peter.pave1dphysik.tu-darmstadt.de
}

For a complete description of the physical properties of low-energy QCD, it might be advantageous to first reformulate QCD in terms of gauge-invariant dynamical variables, before applying any approximation schemes. Using a canonical transformation of the dynamical variables, which Abelianises the non-Abelian Gauss-law constraints to be implemented, such a reformulation can be achieved for QCD. The exact implementation of the Gauss laws reduces the colored spin-1 gluons and spin-1/2 quarks to unconstrained colorless spin- 0 , spin-1, spin-2 and spin-3 glueball fields and colorless Rarita-Schwinger fields respectively. The obtained physical Hamiltonian can then be rewritten into a form, which separates the rotational from the scalar degrees of freedom, and admits a systematic strong-coupling expansion in powers of $\lambda=g^{-2 / 3}$, equivalent to an expansion in the number of spatial derivatives. The leading-order term in this expansion corresponds to non-interacting hybrid-glueballs, whose low-lying masses can be calculated with high accuracy by solving the Schrödinger-equation of the Dirac-Yang-Mills quantum mechanics of spatially constant physical fields (at the moment only for the 2-color case). Due to the presence of classical zero-energy valleys of the chromomagnetic potential for two arbitrarily large classical glueball fields (the unconstrained analogs of the well-known constant Abelian fields), practically all glueball excitation energy is expected to go into the increase of the strengths of these two fields. Higher-order terms in $\lambda$ lead to interactions between the hybrid-glueballs and can be taken into account systematically using perturbation theory in $\lambda$.

Xth Quark Confinement and the Hadron Spectrum

8-12 October 2012

TUM Campus Garching, Munich, Germany

\footnotetext{
* Speaker.
} 


\section{Introduction}

The QCD action

$$
\mathscr{S}[A, \psi, \bar{\psi}]=\int d^{4} x\left[-\frac{1}{4} F_{\mu v}^{a} F^{a \mu v}+\bar{\psi}\left(i \gamma^{\mu} D_{\mu}-m\right) \psi\right]
$$

is invariant under the $S U(3)$ gauge transformations $U[\omega(x)] \equiv \exp \left(i \omega_{a} \tau_{a} / 2\right)$

$$
\psi^{\omega}(x)=U[\omega(x)] \psi(x), \quad A_{a \mu}^{\omega}(x) \tau_{a} / 2=U[\omega(x)]\left(A_{a \mu}(x) \tau_{a} / 2+\frac{i}{g} \partial_{\mu}\right) U^{-1}[\omega(x)] .
$$

Introducing the chromoelectric $E_{i}^{a} \equiv F_{i 0}^{a}$ and chromomagnetic $B_{i}^{a} \equiv \frac{1}{2} \varepsilon_{i j k} F_{j k}^{a}$ and noting that the momenta conjugate to the spatial $A_{a i}$ are $\Pi_{a i}=-E_{a i}$, one obtains the canonical Hamiltonian

$$
H_{C}=\int d^{3} x\left[\frac{1}{2} E_{a i}^{2}+\frac{1}{2} B_{a i}^{2}(A)-g A_{a i} j_{i a}(\psi)+\bar{\psi}\left(\gamma_{i} \partial_{i}+m\right) \psi-g A_{a 0}\left(D_{i}(A)_{a b} E_{b i}-\rho_{a}(\psi)\right)\right],
$$

with the covariant derivative $D_{i}(A)_{a b} \equiv \delta_{a b} \partial_{i}-g f_{a b c} A_{c i}$ in the adjoint representation.

Exploiting the time dependence of the gauge transformations ([L.]) to put (see e.g. [四)

$$
A_{a 0}=0, \quad a=1, . ., 8 \quad \text { (Weyl gauge) }
$$

and quantising the dynamical variables $A_{a i},-E_{a i}, \psi_{\alpha r}$ and $\psi_{\alpha r}^{*}$ in the Schrödinger functional approach by imposing equal-time (anti-) commutation relations (CR), e.g. $-E_{a i}=-i \partial / \partial A_{a i}$, the physical states $\Phi$ have to satisfy both the Schrödinger equation and the Gauss laws

$$
\begin{aligned}
H \Phi & =\int d^{3} x\left[\frac{1}{2} E_{a i}^{2}+\frac{1}{2} B_{a i}^{2}[A]-A_{a i} j_{i a}(\psi)+\bar{\psi}\left(\gamma_{i} \partial_{i}+m\right) \psi\right] \Phi=E \Phi, \\
G_{a}(x) \Phi & =\left[D_{i}(A)_{a b} E_{b i}-\rho_{a}(\psi)\right] \Phi=0, \quad a=1, . ., 8 .
\end{aligned}
$$

The Gauss law operators $G_{a}$ are the generators of the residual time independent gauge transformations in ([L2), satisfying $\left[G_{a}(x), H\right]=0$ and $\left[G_{a}(x), G_{b}(y)\right]=i f_{a b c} G_{c}(x) \delta(x-y)$.

Furthermore, $H$ commutes with the angular momentum operators

$$
J_{i}=\int d^{3} x\left[-\varepsilon_{i j k} A_{a j} E_{a k}+\Sigma_{i}(\psi)+\text { orbital parts }\right], \quad i=1,2,3 .
$$

The matrix element of an operator $O$ is given in the Cartesian form

$$
\left\langle\Phi^{\prime}|O| \Phi\right\rangle \propto \int d A d \bar{\psi} d \psi \Phi^{\prime *}(A, \bar{\psi}, \psi) O \Phi(A, \bar{\psi}, \psi) .
$$

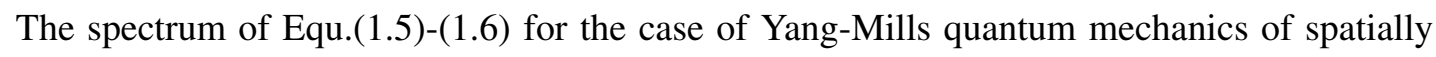
constant gluon fields, has been found in [] ] for $S U(2)$ and in [3] for $S U(3)$, in the context of a weak coupling expansion in $g^{2 / 3}$, using the variational approach with gauge-invariant wave-functionals automatically satisfying (ㄸ.6). The corresponding unconstrained approach, a description in terms of gauge-invariant dynamical variables via an exact implementation of the Gaws laws, has been considered by many authors (o.a. [四, [四]-[四], and references therein) to obtain a non-perturbative description of QCD at low energy, as an alternative to lattice QCD.

I shall first discuss in Section 2 the unphysical, but technically much simpler case of 2-colors, and then show in Section 3 how the results can be generalised to $S U(3)$. 


\section{Unconstrained Hamiltonian formulation of 2-color QCD}

\subsection{Canonical transformation to adapted coordinates}

Point transformation from the $A_{a i}, \psi_{\alpha}$ to a new set of adapted coordinates, the 3 angles $q_{j}$ of an orthogonal matrix $O(q)$, the 6 elements of a pos. definite symmetric $3 \times 3$ matrix $S$, and new $\psi_{\beta}^{\prime}$

$$
A_{a i}(q, S)=O_{a k}(q) S_{k i}-\frac{1}{2 g} \varepsilon_{a b c}\left(O(q) \partial_{i} O^{T}(q)\right)_{b c}, \quad \psi_{\alpha}\left(q, \psi^{\prime}\right)=U_{\alpha \beta}(q) \psi_{\beta}^{\prime},
$$

where the orthogonal $O(q)$ and the unitary $U(q)$ are related via $O_{a b}(q)=\frac{1}{2} \operatorname{Tr}\left(U^{-1}(q) \tau_{a} U(q) \tau_{b}\right)$. Equ. (لـ) is the generalisation of the (unique) polar decomposition of $A$ and corresponds to

$$
\chi_{i}(A)=\varepsilon_{i j k} A_{j k}=0 \quad \text { ("symmetric gauge"). }
$$

Preserving the $\mathrm{CR}$, we obtain the old canonical momenta in terms of the new variables

$$
-E_{a i}(q, S, p, P)=O_{a k}(q)\left[P_{k i}+\varepsilon_{k i l}{ }^{*} D_{l s}^{-1}(S)\left(\Omega_{s j}^{-1}(q) p_{j}+\rho_{s}\left(\psi^{\prime}\right)+D_{n}(S)_{s m} P_{m n}\right)\right] .
$$

In terms of the new canonical variables the Gauss law constraints are Abelianised,

$$
G_{a} \Phi \equiv O_{a k}(q) \Omega_{k i}^{-1}(q) p_{i} \Phi=0 \quad \Leftrightarrow \quad \frac{\delta}{\delta q_{i}} \Phi=0 \quad \text { (Abelianisation), }
$$

and the angular momenta become

$$
J_{i}=\int d^{3} x\left[-2 \varepsilon_{i j k} S_{m j} P_{m k}+\Sigma_{i}\left(\psi^{\prime}\right)+\rho_{i}\left(\psi^{\prime}\right)+\text { orbital parts }\right] .
$$

Equ.(2. (2) identifies the $q_{i}$ with the gauge angles and $S$ and $\psi^{\prime}$ as the physical fields. Furthermore, from Equ.(2.5) follows that the $S$ are colorless spin- 0 and spin-2 glueball fields, and the $\psi^{\prime}$ colorless reduced quark fields of spin- 0 and spin- 1 . Hence the gauge reduction corresponds to the conversion "color $\rightarrow$ spin". The obtained unusual spin-statistics relation is specific to SU(2).

\subsection{Physical quantum Hamiltonian}

According to the general scheme [四], the correctly ordered physical quantum Hamiltonian in terms of the physical variables $S_{i k}(\mathbf{x})$ and the canonically conjugate $P_{i k}(\mathbf{x}) \equiv-i \delta / \delta S_{i k}(\mathbf{x})$ reads [[]]

$$
\begin{array}{r}
H(S, P)=\frac{1}{2} \mathscr{J}^{-1} \int d^{3} \mathbf{x} P_{a i} \mathscr{J} P_{a i}+\frac{1}{2} \int d^{3} \mathbf{x}\left[B_{a i}^{2}(S)-S_{a i} j_{i a}\left(\psi^{\prime}\right)+\bar{\psi}^{\prime}\left(\gamma_{i} \partial_{i}+m\right) \psi^{\prime}\right] \\
-\mathscr{J}^{-1} \int d^{3} \mathbf{x} \int d^{3} \mathbf{y}\left\{\left(D_{i}(S)_{m a} P_{i m}+\rho_{a}\left(\psi^{\prime}\right)\right)(\mathbf{x}) \mathscr{J}\right. \\
\left.\left\langle\left.\mathbf{x} a\right|^{*} D^{-2}(S) \mid \mathbf{y} b\right\rangle\left(D_{j}(S)_{b n} P_{n j}+\rho_{b}\left(\psi^{\prime}\right)\right)(\mathbf{y})\right\}
\end{array}
$$

with the Faddeev-Popov (FP) operator

$$
{ }^{*} D_{k l}(S) \equiv \varepsilon_{k m i} D_{i}(S)_{m l}=\varepsilon_{k l i} \partial_{i}-g\left(S_{k l}-\delta_{k l} \mathrm{tr} S\right),
$$

and the Jacobian $\left.\mathscr{J} \equiv \operatorname{det}\right|^{*} D \mid$. The matrix element of a physical operator $\mathrm{O}$ is given by

$$
\left\langle\Psi^{\prime}|O| \Psi\right\rangle \propto \int_{\text {S pos.def. }} \int_{\bar{\psi}^{\prime}, \psi^{\prime}} \prod_{\mathbf{x}}\left[d S(\mathbf{x}) d \bar{\psi}^{\prime}(\mathbf{x}) d \psi^{\prime}(\mathbf{x})\right] \mathscr{J} \Psi^{\prime *}\left[S, \bar{\psi}^{\prime}, \psi^{\prime}\right] O \Psi\left[S, \bar{\psi}^{\prime}, \psi^{\prime}\right] .
$$

The inverse of the FP operator and hence the physical Hamiltonian can be expanded in the number of spatial derivatives, equivalent to a strong coupling expansion in $\lambda=g^{-2 / 3}$. 
2.3 Coarse-graining and strong coupling expansion of the physical Hamiltonian in $\lambda=g^{-2 / 3}$

Introducing an UV cutoff $a$ by considering an infinite spatial lattice of granulas $G(\mathbf{n}, a)$ at $\mathbf{x}=a \mathbf{n}\left(\mathbf{n} \in Z^{3}\right)$ and averaged variables

$$
S(\mathbf{n}):=\frac{1}{a^{3}} \int_{G(\mathbf{n}, a)} d \mathbf{x} S(\mathbf{x}),
$$

and discretised spatial derivatives, the expansion of the Hamiltonian in $\lambda=g^{-2 / 3}$ can be written

$$
H=\frac{g^{2 / 3}}{a}\left[\mathscr{H}_{0}+\lambda \sum_{\alpha} \mathscr{V}_{\alpha}^{(\partial)}+\lambda^{2}\left(\sum_{\beta} \mathscr{V}_{\beta}^{(\Delta)}+\sum_{\gamma} \mathscr{V}_{\gamma}^{(\partial \partial \neq \Delta)}\right)+\mathscr{O}\left(\lambda^{3}\right)\right] .
$$

The "free" Hamiltonian $H_{0}=\left(g^{2 / 3} / a\right) \mathscr{H}_{0}+H_{m}=\sum_{\mathbf{n}} H_{0}^{Q M}(\mathbf{n})$ is the sum of the Hamiltonians of Dirac-Yang-Mills quantum mechanics of constant fields in each box, and the interaction terms $\mathscr{V}^{(\partial)}, \mathscr{V}^{(\Delta)}, .$. leading to interactions between the granulas.

\subsection{Zeroth-order: Dirac-Yang-Mills Quantum mechanics of spatially constant fields}

Transforming to the intrinsic system of the symmetric tensor $S$, with Jacobian $\sin \beta \prod_{i<j}\left(\phi_{i}-\phi_{j}\right)$,

$$
S=R^{T}(\alpha, \beta, \gamma) \operatorname{diag}\left(\phi_{1}, \phi_{2}, \phi_{3}\right) R(\alpha, \beta, \gamma), \quad \psi_{L, R}^{\prime(i)}=R_{i j}^{T} \widetilde{\psi}_{L, R}^{(j)}, \quad \psi_{L, R}^{\prime(0)}=\widetilde{\psi}_{L, R}^{(0)},
$$

the "free" Hamiltonian in each box (volume $V$ ) takes the form [Q]

$$
H_{0}^{Q M}=\frac{g^{2 / 3}}{V^{1 / 3}}\left[\mathscr{H}^{G}+\mathscr{H}^{D}+\mathscr{H}^{C}\right]+\frac{1}{2} m\left[\left(\widetilde{\psi}_{L}^{(0) \dagger} \widetilde{\psi}_{R}^{(0)}+\sum_{i=1}^{3} \widetilde{\psi}_{L}^{(i) \dagger} \widetilde{\psi}_{R}^{(i)}\right)+h . c .\right],
$$

with the glueball part $\mathscr{H}^{G}$, the minimal-coupling $\mathscr{H}^{D}$, and the Coulomb-potential-type part $\mathscr{H}^{C}$

$$
\begin{aligned}
& \mathscr{H}^{G}=\frac{1}{2} \sum_{i j k}^{\text {cyclic }}\left(-\frac{\partial^{2}}{\partial \phi_{i}^{2}}-\frac{2}{\phi_{i}^{2}-\phi_{j}^{2}}\left(\phi_{i} \frac{\partial}{\partial \phi_{i}}-\phi_{j} \frac{\partial}{\partial \phi_{j}}\right)+\left(\xi_{i}-\widetilde{J}_{i}^{Q}\right)^{2} \frac{\phi_{j}^{2}+\phi_{k}^{2}}{\left(\phi_{j}^{2}-\phi_{k}^{2}\right)^{2}}+\phi_{j}^{2} \phi_{k}^{2}\right) \\
& \mathscr{H}^{D}=\frac{1}{2}\left(\phi_{1}+\phi_{2}+\phi_{3}\right)\left(\widetilde{N}_{L}^{(0)}-\widetilde{N}_{R}^{(0)}\right)+\frac{1}{2} \sum_{i j k}^{\text {cyclic }}\left(\phi_{i}-\left(\phi_{j}+\phi_{k}\right)\right)\left(\widetilde{N}_{L}^{(i)}-\widetilde{N}_{R}^{(i)}\right) \\
& \mathscr{H}^{C}=\sum_{i j k}^{\text {cyclic }} \frac{\widetilde{\rho}_{i}\left(\xi_{i}-\widetilde{J}_{i}^{Q}+\widetilde{\rho}_{i}\right)}{\left(\phi_{j}+\phi_{k}\right)^{2}} \\
& \quad J_{i}=R_{i j}(\chi) \xi_{j}, \quad\left[J_{i}, H\right]=0
\end{aligned}
$$

and the total spin

The matrix elements become

$$
\left\langle\Phi_{1}|\mathscr{O}| \Phi_{2}\right\rangle=\int d \alpha \sin \beta d \beta d \gamma \int_{0<\phi_{1}<\phi_{2}<\phi_{3}}^{d} \underset{\phi_{1}}{ } d \phi_{2} d \phi_{3}\left(\phi_{1}^{2}-\phi_{2}^{2}\right)\left(\phi_{2}^{2}-\phi_{3}^{2}\right)\left(\phi_{3}^{2}-\phi_{1}^{2}\right) \int d \bar{\psi}^{\prime} d \psi^{\prime} \Phi_{1}^{*} \mathscr{O} \Phi_{2} .
$$

The 1.h.s. of Fig. 1 shows the $0^{+}$energy spectrum of the lowest pure-gluon $(\mathrm{G})$ and quark-gluon (QG) cases for one quark-flavor which can be calculated with high accuracy using the variational approach. The energies of the quark-gluon ground state and the sigma-antisigma excitation are lower than that of the lowest pure-gluon state. This is due to a large negative contribution from $\left\langle\mathscr{H}^{D}\right\rangle$, in addition to the large positive $\left\langle\mathscr{H}^{G}\right\rangle$, while $\left\langle\mathscr{H}^{C}\right\rangle \simeq 0$ (see [9] for details).

Furthermore, as a consequence of the zero-energy valleys " $\phi_{1}=\phi_{2}=0, \phi_{3}$ arbitrary" of the classical magnetic potential $B^{2}=\phi_{2}^{2} \phi_{3}^{2}+\phi_{3}^{2} \phi_{1}^{2}+\phi_{1}^{2} \phi_{2}^{2}$, practically all glueball excitation-energy results from an increase of expectation value of the "constant Abelian field" $\phi_{3}$ as shown for the

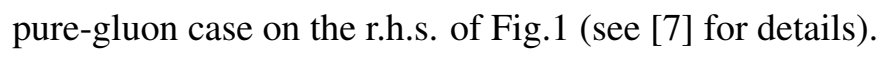



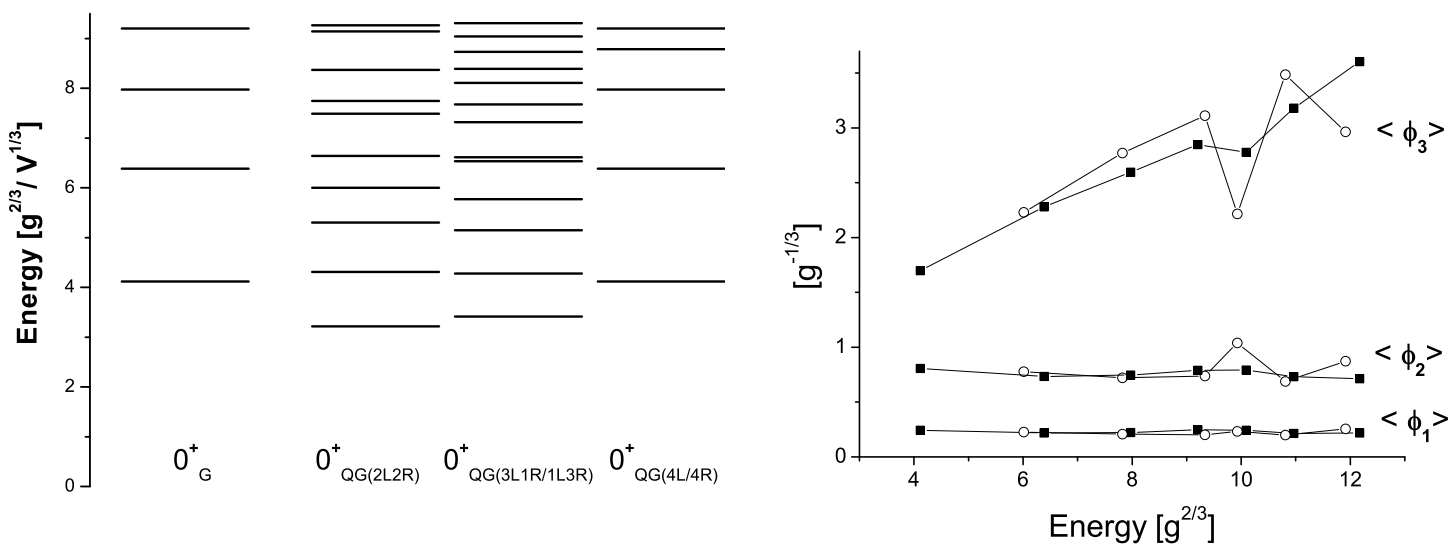

Figure 1: L.h.s.: Lowest energy levels for the pure-gluon $(\mathrm{G})$ and the quark-gluon case (QG) for 2-colors and one quark flavor. The energies of the quark-gluon ground state and the sigma-antisigma excitation are lower than that of the lowest pure-gluon state. R.h.s. (for pure-gluon case and setting $V \equiv 1$ ): $\left\langle\phi_{3}\right\rangle$ is raising with increasing excitation, whereas $\left\langle\phi_{1}\right\rangle$ and $\left\langle\phi_{2}\right\rangle$ are practically constant, independent of whether spin- 0 (dark boxes) or spin-2 states (open circles).

\subsection{Perturbation theory in $\lambda$ and coupling constant renormalisation in the IR}

Including the interactions $\mathscr{V}^{(\partial)}, \mathscr{V}^{(\Delta)}$ using 1st and 2nd order perturbation theory in $\lambda=g^{-2 / 3}$ give the result $[[]]$ (for pure-gluon case and only including spin-0 fields in a first approximation)

$$
\begin{aligned}
E_{\mathrm{vac}}^{+} & =\mathscr{N} \frac{g^{2 / 3}}{a}\left[4.1167+29.894 \lambda^{2}+\mathscr{O}\left(\lambda^{3}\right)\right], \\
E_{1}^{(0)+}(k)-E_{\mathrm{vac}}^{+} & =\left[2.270+13.511 \lambda^{2}+\mathscr{O}\left(\lambda^{3}\right)\right] \frac{g^{2 / 3}}{a}+0.488 \frac{a}{g^{2 / 3}} k^{2}+\mathscr{O}\left(\left(a^{2} k^{2}\right)^{2}\right),
\end{aligned}
$$

for the energy of the interacting glueball vacuum and the spectrum of the interacting spin-0 glueball. Lorentz invariance demands $E=\sqrt{M^{2}+k^{2}} \simeq M+\frac{1}{2 M} k^{2}$, which is violated in this 1st approximation by a factor of 2 . In order to get a Lorentz invariant result, $J=L+S$ states should be considered including also spin-2 states and the general $\mathscr{V}^{(\partial \partial)}$.

Independence of the physical glueball mass

$$
M=\frac{g_{0}^{2 / 3}}{a}\left[\mu+c g_{0}^{-4 / 3}\right]
$$

of box size $a$, one obtains

$$
\gamma\left(g_{0}\right) \equiv a \frac{d}{d a} g_{0}(a)=\frac{3}{2} g_{0} \frac{\mu+c g_{0}^{-4 / 3}}{\mu-c g_{0}^{-4 / 3}}
$$

which vanishes for $g_{0}=0$ (pert. fixed point) or $g_{0}^{4 / 3}=-c / \mu$ (IR fixed point, if $c<0$ ). For $c>0$

$$
\text { for } c>0: \quad g_{0}^{2 / 3}(M a)=\frac{M a}{2 \mu}+\sqrt{\left(\frac{M a}{2 \mu}\right)^{2}-\frac{c}{\mu}}, \quad a>a_{c}:=2 \sqrt{c \mu} / M
$$

My (incomplete) result $c_{1}^{(0)} / \mu_{1}^{(0)}=5.95$ suggests, that no IR fixed points exist. critical coupling $\left.g_{0}^{2}\right|_{c}=14.52$ and $a_{c} \sim 1.4 \mathrm{fm}$ for $M \sim 1.6 \mathrm{GeV}$. 


\section{Symmetric gauge for $\mathrm{SU}(3)$}

Using the idea of minimal embedding of $s u(2)$ in $s u(3)$ by Kihlberg and Marnelius [ [G]

$$
\begin{array}{ll}
\tau_{1}:=\lambda_{7}=\left(\begin{array}{ccc}
0 & 0 & 0 \\
0 & 0 & -i \\
0 & i & 0
\end{array}\right) & \tau_{2}:=-\lambda_{5}=\left(\begin{array}{ccc}
0 & 0 & i \\
0 & 0 & 0 \\
-i & 0 & 0
\end{array}\right) \\
\tau_{4}:=\lambda_{6}=\left(\begin{array}{lll}
0 & 0 & 0 \\
0 & 0 & 1 \\
0 & 1 & 0
\end{array}\right) & \tau_{5}:=\lambda_{4}=\left(\begin{array}{lll}
0 & 0 & 1 \\
0 & 0 & 0 \\
1 & 0 & 0
\end{array}\right) \quad \tau_{6}:=\lambda_{2}=\left(\begin{array}{ccc}
0 & -i & 0 \\
i & 0 & 0 \\
0 & 0 & 0
\end{array}\right) \\
\tau_{7}:=\lambda_{3}=\left(\begin{array}{ccc}
1 & 0 & 0 \\
0 & -1 & 0 \\
1 & 0 & 0 \\
0 & 0 & 0
\end{array}\right) & \tau_{8}:=\lambda_{8}=\frac{1}{\sqrt{3}}\left(\begin{array}{ccc}
1 & 0 & 0 \\
0 & 1 & 0 \\
0 & 0 & -2
\end{array}\right)
\end{array}
$$

such that the corresponding non-trivial non-vanishing structure constants, $\left[\frac{\tau_{a}}{2}, \frac{\tau_{b}}{2}\right]=i c_{a b c} \frac{\tau_{c}}{2}$, have at least one index $\in\{1,2,3\}$, the symmetric gauge, Equ.([2.2), can be generalised to $S U(3)$ [[1], [0]],

$$
\left.\chi_{a}(A)=\sum_{b=1}^{8} \sum_{i=1}^{3} c_{a b i} A_{b i}=0, \quad a=1, \ldots, 8 \quad \text { ("symmetric gauge" for } \mathrm{SU}(3)\right) .
$$

Carrying out the coordinate transformation [ए]]

$$
\begin{aligned}
& A_{a k}\left(q_{1}, . ., q_{8}, \widehat{S}\right)=O_{a \hat{a}}(q) \widehat{S}_{\hat{a} k}-\frac{1}{2 g} c_{a b c}\left(O(q) \partial_{k} O^{T}(q)\right)_{b c}, \quad \psi_{\alpha}\left(q_{1}, . ., q_{8}, \psi^{R S}\right)=U_{\alpha \hat{\beta}}(q) \psi_{\hat{\beta}}^{R S} \\
& \widehat{S}_{\hat{a} k} \equiv\left(\begin{array}{c}
S_{i k} \\
\bar{S}_{A k}
\end{array}\right)=\left(\begin{array}{ccc}
\multicolumn{3}{c}{S_{i k} \text { pos. def. }} \\
X_{0} & X_{3}-W_{3} & X_{2}+W_{2} \\
X_{3}+W_{3} & W_{0} & X_{1}-W_{1} \\
X_{2}-W_{2} & X_{1}+W_{1} & W_{0} \\
-\frac{\sqrt{3}}{2} Y_{1}-\frac{1}{2} W_{1} & \frac{\sqrt{3}}{2} Y_{2}-\frac{1}{2} W_{2} & W_{3} \\
-\frac{\sqrt{3}}{2} W_{1}-\frac{1}{2} Y_{1} & \frac{\sqrt{3}}{2} W_{2}-\frac{1}{2} Y_{2} & Y_{3}
\end{array}\right), \quad c_{\hat{a} \hat{b} k} \widehat{S}_{\hat{b} k}=0,
\end{aligned}
$$

an unconstrained Hamiltonian formulation of QCD can be obtained. The existence and uniqueness of (B.3) can be investigated by solving the 16 equs.

$$
\widehat{S}_{\hat{a} i} \widehat{S}_{\hat{a} j}=A_{a i} A_{a j} \text { (6 equs.) } \wedge d_{\hat{a} \hat{b} \hat{c}} \widehat{S}_{\hat{a} i} \widehat{S}_{\hat{b} j} \widehat{S}_{\hat{c} k}=d_{a b c} A_{a i} A_{b j} A_{c k}(10 \text { equs.) }
$$

for the 16 components of $\widehat{S}$ in terms of 24 given components A.

Analysing the Gauss law operators and the unconstrained angular momentum operators in terms of the new variables in analogy to the 2-color case, it can be shown that the original constrained 24 colored spin- 1 gluon fields $A$ and the 12 colored spin-1/2 quark fields $\psi$ (per flavor) reduce to 16 physical colorless spin- 0 , spin- 1 , spin- 2 , and spin- 3 glueball fields (the 16 components of $\widehat{S}$ ) and a colorless spin-3/2 Rarita-Schwinger field $\psi^{R S}$ (per flavor), respectively. As for the 2color case, the gauge reduction converts color $\rightarrow$ spin, which might have important consequences for low energy Spin-Physics. In terms of the colorless Rarita-Schwinger fields the $\Delta^{++}(3 / 2)$ could have the spin content $(+3 / 2,+1 / 2,-1 / 2)$ in accordance with the Spin-Statistics-Theorem. 
Transforming to the intrinsic system of the embedded upper part $\mathrm{S}$ of $\widehat{S}$ (see [प्ठ] for details)

$$
S=R^{T}(\alpha, \beta, \gamma) \operatorname{diag}\left(\phi_{1}, \phi_{2}, \phi_{3}\right) R(\alpha, \beta, \gamma), \wedge X_{i} \rightarrow x_{i}, Y_{i} \rightarrow y_{i}, . . \wedge \psi^{R S} \rightarrow \widetilde{\psi}^{R S},
$$

one finds that the magnetic potential $B^{2}$ has the zero-energy valleys ("constant Abelian fields")

$$
B^{2}=0: \phi_{3} \text { and } y_{3} \text { arbitrary } \wedge \text { all others zero }
$$

Hence, practically all glueball excitation-energy should result from an increase of expectation values of these two "constant Abelian fields", in analogy to $S U(2)$. Furthermore, at the bottom of the valleys the important minimal-coupling-interaction of $\widetilde{\psi}^{R S}$ (analogous to (2.14)) becomes diagonal

$$
\mathscr{H}_{\text {diag }}^{D}=\frac{1}{2} \widetilde{\psi}_{L}^{\left(1, \frac{1}{2}\right) \dagger}\left[\left(\phi_{3} \lambda_{3}+y_{3} \lambda_{8}\right) \otimes \sigma_{3}\right] \widetilde{\psi}_{L}^{\left(1, \frac{1}{2}\right)}-\frac{1}{2} \widetilde{\psi}_{R}^{\left(\frac{1}{2}, 1\right) \dagger}\left[\sigma_{3} \otimes\left(\phi_{3} \lambda_{3}+y_{3} \lambda_{8}\right)\right] \widetilde{\psi}_{R}^{\left(\frac{1}{2}, 1\right)} .
$$

Due to the difficulty of the FP-determinant (see [ए]]), precise calculations are not possible yet. Note, however, that in one spatial dimension the symmetric gauge for SU(3) reduces to

$$
A^{(1 d)}=\left(\begin{array}{lll}
0 & 0 & A_{13} \\
0 & 0 & A_{23} \\
0 & 0 & A_{33} \\
0 & 0 & A_{43} \\
0 & 0 & A_{53} \\
0 & 0 & A_{63} \\
0 & 0 & A_{73} \\
0 & 0 & A_{83}
\end{array}\right) \rightarrow \widehat{S}^{(1 d)}=\widehat{S}_{\text {intrinsic }}^{(1 d)}=\left(\begin{array}{ccc}
0 & 0 & 0 \\
0 & 0 & 0 \\
0 & 0 & \phi_{3} \\
0 & 0 & 0 \\
0 & 0 & 0 \\
0 & 0 & 0 \\
0 & 0 & 0 \\
0 & 0 & y_{3}
\end{array}\right)
$$

which consistently reduces the Equ.(B.4) for given $A_{3}$ to

$$
\phi_{3}^{2}+y_{3}^{2}=A_{a 3} A_{a 3} \quad \wedge \quad \phi_{3}^{2} y_{3}-3 y_{3}^{3}=d_{a b c} A_{a 3} A_{b 3} A_{c 3}
$$

with 6 solutions separated by zero-lines of the FP-determinant ("Gribov-horizons"). Exactly one solution exists in the "fundamental domain" $0<\phi_{3}<\infty \wedge \phi_{3} / \sqrt{3}<y_{3}<\infty$, and we can replace

$$
\int_{-\infty}^{+\infty} \prod_{a=1}^{8} d A_{a 3} \rightarrow \int_{0}^{\infty} d \phi_{3} \int_{\phi_{3} / \sqrt{3}}^{\infty} d y_{3} \phi_{3}^{2}\left(\phi_{3}^{2}-3 y_{3}^{2}\right)^{2} \propto \int_{0}^{\infty} r d r \int_{\pi / 6}^{\pi / 2} d \psi \cos ^{2}(3 \psi) .
$$

For two spatial dimensions, one can show that (putting in Equ.(B.3) $W_{1} \equiv X_{1}, W_{2} \equiv-X_{2}$ )

$$
A^{(2 d)}=\left(\begin{array}{lll}
A_{11} & A_{12} & 0 \\
A_{21} & A_{22} & 0 \\
A_{31} & A_{32} & 0 \\
A_{41} & A_{42} & 0 \\
A_{51} & A_{52} & 0 \\
A_{61} & A_{62} & 0 \\
A_{71} & A_{72} & 0 \\
A_{81} & A_{82} & 0
\end{array}\right) \rightarrow \widehat{S}_{\text {intrinsic }}^{(2 d)}=\left(\begin{array}{ccc}
\phi_{1} & 0 & 0 \\
0 & \phi_{2} & 0 \\
0 & 0 & 0 \\
0 & x_{3} & 0 \\
x_{3} & 0 & 0 \\
2 x_{2} & 2 x_{1} & 0 \\
-\frac{\sqrt{3}}{2} y_{1}-\frac{1}{2} x_{1} & \frac{\sqrt{3}}{2} y_{2}+\frac{1}{2} x_{2} & 0 \\
-\frac{\sqrt{3}}{2} y_{1}+\frac{1}{2} x_{1} & -\frac{\sqrt{3}}{2} y_{2}+\frac{1}{2} x_{2} & 0
\end{array}\right)
$$

consistently reduces (B.4) to a system of 7 equs. for 8 physical fields (incl. rot.-angle $\gamma$ ), which, adding as an 8 th equ. $\left(d_{\hat{a} \hat{b} \hat{c}} \widehat{S}_{\hat{b} 1} \widehat{S}_{\hat{c} 2}\right)^{2}=\left(d_{a b c} A_{b 1} A_{c 2}\right)^{2}$, can be solved numerically for randomly generated $A^{(2 d)}$, again yielding solutions separated by horizons. Restricting to a fundamental domain

$$
\int_{-\infty}^{+\infty} \prod_{a, b=1}^{8} d A_{a 1} d A_{b 2} \rightarrow \int d \gamma \int_{0<\phi_{1}<\phi_{2}<\infty} d \phi_{1} d \phi_{2}\left(\phi_{1}-\phi_{2}\right) \int_{R_{1}\left(\phi_{1}, \phi_{2}\right)} d x_{1} d x_{2} d x_{3} \int_{R_{2}\left(x_{1}, x_{2}, x_{3}, \phi_{1}, \phi_{2}\right)} d y_{1} d y_{2} \mathscr{J}
$$


Due to the difficulty of the FP-determinant, I have, however, not yet succeeded in a satisfactory description of the regions $R_{1}$ and $R_{2}$. For the general case of three dimensions, I have found several solutions of the Equ.(B.4) numerically for a randomly generated $A$, but to write the corresponding unconstrained integral over a fundamental domain is a difficult, but I think solvable, future task.

\section{Conclusions}

Using a canonical transformation of the dynamical variables, which Abelianises the nonAbelian Gauss-law constraints to be implemented, a reformulation of QCD in terms of gauge invariant dynamical variables can be achieved. The exact implementation of the Gauss laws reduces the colored spin- 1 gluons and spin-1/2 quarks to unconstrained colorless spin- 0 , spin- 1 , spin- 2 and spin-3 glueball fields and colorless Rarita-Schwinger fields respectively. The obtained physical Hamiltonian admits a systematic strong-coupling expansion in powers of $\lambda=g^{-2 / 3}$, equivalent to an expansion in the number of spatial derivatives. The leading-order term in this expansion corresponds to non-interacting hybrid-glueballs, whose low-lying masses can be calculated with high accuracy (at the moment only for the unphysical, but technically much simpler 2-color case) by solving the Schrödinger-equation of Dirac-Yang-Mills quantum mechanics of spatially constant fields. Higher-order terms in $\lambda$ lead to interactions between the hybrid-glueballs and can be taken into account systematically, using perturbation theory in $\lambda$, allowing for the study of the difficult questions of Lorentz invariance and coupling constant renormalisation in the IR.

\section{References}

[1] N.H. Christ and T.D. Lee, Operator ordering and Feynman rules in gauge theories, Phys. Rev. D 22 (1980) 939.

[2] M. Lüscher and G. Münster, Weak coupling expansion of the low-lying energy values in the $S U(2)$ gauge theory on a torus, Nucl. Phys. B 232 (1984) 445.

[3] P. Weisz and V. Ziemann, Weak coupling expansion of the low-lying energy values in SU(3) gauge theory on a torus, Nucl. Phys. B 284 (1987) 157.

[4] A. Kihlberg and R. Marnelius, Properties of Yang-Mills theories with gauge-fixing conditions on the field strength, Phys. Rev. D 26 (1982) 2003.

[5] B. Dahmen and B. Raabe, Unconstrained SU(2) and SU(3) Yang-Mills classical mechanics, Nucl. Phys. B 384 (1992) 352.

[6] A.M. Khvedelidze and H.-P. Pavel, Unconstrained Hamiltonian formulation of SU(2) gluodynamics, Phys. Rev. D 59 (1999) 105017 [hep-th/9808102].

[7] H.-P. Pavel, SU(2) Yang-Mills quantum mechanics of spatially constant fields, Phys. Lett. B 648 (2007) 97 [hep-th/ 0701283$].$

[8] H.-P. Pavel, Expansion of the Yang-Mills Hamiltonian in spatial derivatives and glueball spectrum, Phys. Lett. B 685 (2010) 353 [arXiv: 0912.5465 [hep-th] ].

[9] H.-P. Pavel, SU(2) Dirac-Yang-Mills quantum mechanics of spatially constant quark and gluon fields, Phys. Lett. B 700 (2011) 265 [arXiv:1104.1576 [hep-th] ].

[10] H.-P. Pavel, Unconstrained Hamiltonian formulation of low energy SU(3) Yang-Mills quantum theory, arXiv: 1205.2237 [hep-th] (2012). 